\title{
De l'agrégation à la floculation des asphaltènes, une description structurale par diffusion de rayonnement
}

\author{
D. Fenistein, L. Barré', D. Frot ${ }^{1}$ \\ 1 Institut français du pétrole, 1 et 4, avenue de Bois-Préau, 92852 Rueil-Malmaison Cedex - France \\ e-mail : didier.frot@ifp.fr
}

\begin{abstract}
Résumé - L'étude par diffusion de rayonnement (rayons X, neutrons et lumière) d'asphaltènes dans des mélanges de toluène et d'heptane en proportion relative croissante permet de caractériser leur structure d'agrégation. Dans un solvant contenant peu d'heptane, les agrégats d'asphaltènes sont fractals et solvatés. Leur cinétique d'agrégation est compatible avec les prévisions du modèle théorique d'agrégation RLCA (Reaction Limited Cluster Aggregation). Pour des proportions d'heptane supérieures, les asphaltènes s'arrangent en structures compactes et de très grande taille. Nous associons cette transition entre un processus d'agrégation solvatée et une compaction au phénomène de floculation des asphaltènes. L'étude par diffusion des rayons X aux très petits angles du RSV (résidu sous vide) Safaniya montre que ces agrégats compacts d'asphaltènes floculés peuvent exister dans certains fluides industriels. Mots-clés : précipitation d'asphaltènes, agrégation, floculation, structures solvatées (fractales) et compactes, diffusion de rayonnement.
\end{abstract}

\footnotetext{
Abstract - From Aggregation to Flocculation of Asphaltenes, a Structural Description by Radiation Scattering Techniques - The study, using radiation scattering techniques (X-rays, neutrons, light) of asphaltenes in toluene and heptane mixtures, reveals their structure of aggregation versus the heptane/toluene ratio. In a solvent containing little heptane, the aggregates of asphaltenes are fractal and solvated. Their kinetics of aggregation is compatible with the forecasts model of aggregation RLCA (Reaction Limited Cluster Aggregation). For higher proportions of heptane the asphaltenes arrange in compact structures of very big sizes. We associate this transition, between a solvated aggregation and a compaction process, to the asphaltene flocculation phenomenon. The study, using the very small angle $X$ ray scattering technique, of the Safaniya vaccum residue shows that these compact asphaltene aggregates may exist in some industrial fluids.

Keywords: asphaltene precipitation, aggregation, flocculation, solvated (fractal) and compact structures, radiation scattering techniques.
} 


\section{INTRODUCTION}

Les asphaltènes constituent la fraction lourde d'un produit pétrolier définie comme insoluble dans un excès d'alcane léger (30 volumes d'heptane pour la norme française AFNOR T60-115). Ils sont composés [1] de parties aromatiques polycondensées peu étendues (de trois à cinq noyaux aromatiques) liées à des chaînes aliphatiques et/ou naphténiques qui s'arrangent, d'après l'image proposée par Yen [2], en micelles de quelques dizaines d'angströms.

L'une des principales motivations de l'étude des asphaltènes est la compréhension de leur capacité à floculer. La stabilité des asphaltènes en fonction des conditions extérieures (température, pression et composition chimique du solvant) a été largement étudiée ces dernières années [3, 4]. On définit le seuil de floculation comme la quantité minimale d'heptane nécessaire à la précipitation des premiers asphaltènes. La détermination et la prévision du seuil de floculation sont une préoccupation majeure de l'industrie pétrolière [5]. La floculation des asphaltènes conduit à la formation d'agrégats asphalténiques de grande taille (de l'ordre du micron) qui précipitent. Mais la connaissance de leur structure, de l'organisation des molécules au sein des agrégats, reste limitée.

Une agrégation progressive fractale et solvatée des asphaltènes est observée à l'approche du seuil de floculation [6-12] et comparée aux modèles théoriques d'agrégation des colloïdes (DLCA, Diffusion Limited Cluster Aggregation, ou RLCA) [13-17]. Ces modèles prédisent la structure et la cinétique d'agrégation de particules qui s'agrègent sous l'effet d'un potentiel d'interaction attractif de portée limitée. Les particules colloïdales diffusent et, à terme, se rencontrent. Lorsque le potentiel d'interaction est tel que chaque contact entre particules entraîne leur liaison, le processus d'agrégation qui s'ensuit est appelé «DLCA»; lorsqu'il existe une probabilité (différente de 1) pour qu'un contact entre particules entraîne leur liaison, l'agrégation est appelée «RLCA». Les agrégats diffusant eux-mêmes s'agrègent aussi avec d'autres particules ou agrégats. Cependant, au seuil de floculation, la sédimentation rapide des asphaltènes floculés est peu compatible avec les structures solvates (DLCA ou RLCA) mesurées à l'approche du seuil de floculation.

Nous proposons ici une étude visant à décrire le passage du seuil de floculation en termes de structure d'agrégation. Nous étudions les asphaltènes en solution dans des mélanges de bon solvant (toluène) et de floculant (heptane), ce qui permet de contrôler la qualité du solvant. Nous utiliserons les techniques de diffusion de rayonnement particulièrement adaptées à l'étude de tels systèmes colloïdaux. Par diffusion des rayons X (SAXS, Small Angle X-ray Scattering) et des neutrons (SANS, Small Angle Neutron Scattering), nous pourrons explorer la taille (de quelques dizaines à quelques centaines d'angströms) des agrégats, correspondant plutôt au début de l'agrégation des asphaltènes. L'échelle de taille accessible en diffusion de la lumière (de quelques centaines d'angströms à quelques microns) permet l'étude de la cinétique d'agrégation des asphaltènes au passage du seuil de floculation. L'utilisation d'un montage spécifique (IFPSematech [18]) de diffusion dynamique de la lumière, analysant l'intensité de la lumière rétrodiffusée par l'échantillon plutôt que celle diffusée vers l'avant, permet l'étude des milieux noirs et concentrés en asphaltènes.

\section{MÉTHODES EXPÉRIMENTALES : DES OUTILS POUR MILIEUX OPAQUES}

\subsection{Diffusion statique des rayons $X$}

Le montage de diffusion des rayons $\mathrm{X}$ aux petits angles (SAXS) se trouve à l'IFP. Il permet d'accéder à des vecteurs de diffusion allant de $10^{-2}$ à $0,2 \AA^{-1}$. Le rayonnement polychromatique, produit par une anode tournante en cuivre (RU 200), est filtré par un filtre en nickel. La longueur d'onde du faisceau sortant est celle de la raie $\mathrm{K}_{\alpha}$ du cuivre. Les échantillons sont placés dans des capillaires cylindriques de $2 \mathrm{~mm}$ de diamètre, en verre.

Le montage aux très petits angles (USAXS, Ultra Small Angle X-ray Scattering) se trouve au Laboratoire de chimie moléculaire du CEA-Saclay [19]. C'est une caméra «BonzeHart », constituée de deux cristaux à gorge, qui permet de mesurer des vecteurs de diffusion allant de $5 \cdot 10^{-4}$ à $5 \cdot 10^{-2} \AA^{-1}$. Le rayonnement est produit selon le même principe que pour le montage SAXS. La déconvolution des spectres USAXS se fait à l'aide de la méthode de Lake [20].

\subsection{Diffusion statique des neutrons}

Les expériences de diffusion des neutrons (SANS) sont réalisées au laboratoire Léon-Brillouin au CEA-Saclay sur le montage PACE. La gamme de vecteurs de diffusion accessible s'étend de $3 \cdot 10^{-3}$ à $0,2 \AA^{-1}$. Les échantillons sont placés dans des cellules Hellma parallélépipédiques d'épaisseur égale à $2 \mathrm{~mm}$. Les longueurs d'onde entre 4 et 20 Å sont sélectionnées avec une résolution de $10 \%$.

Les spectres de diffusion des neutrons et des rayons $\mathrm{X}$ sont exprimés en échelle absolue.

\subsection{Diffusion dynamique de la lumière}

Les mesures de diffusion de la lumière sont faites à l'IFP. Le faisceau lumineux est produit par un laser He-Ne dont la puissance maximale atteint $25 \mathrm{~mW}$. Le domaine de taille 
accessible s'étend de quelques centaines d'angströms à quelques microns. Le détecteur enregistre l'intensité du rayonnement rétrodiffusé par l'échantillon. Ce dispositif permet au faisceau de ne traverser qu'une faible épaisseur de l'échantillon (sans diffusion multiple), de n'être que peu absorbé (sans augmentation locale de la température) et d'enregistrer le signal diffusé par une solution concentrée en asphaltènes.

\subsection{Ultracentrifugation}

Le montage d'ultracentrifugation est un modèle Beckman E qui se trouve au Laboratoire de chimie des polymères inorganiques de l'université Pierre-et-Marie-Curie. Il est constitué d'un rotor métallique au sein d'une chambre sous vide qui permet de limiter les frictions. La distance à l'axe et la taille de la cellule sont telles que $r_{\min }=5,7 \mathrm{~cm}$ et $r_{\max }=$ $12,7 \mathrm{~cm}$. Nous avons séparé une solution diluée (facteur volumique $\Phi \approx 0,03$ ) d'asphaltènes Safaniya dans le toluène par ultracentrifugation. La vitesse de rotation appliquée, $\omega \approx$ $40000 \mathrm{tr} / \mathrm{min}$ pendant 10 heures, correspond à une accélération de d'environ $10^{5} \mathrm{~g}$. Six fractions sont extraites, pesées, leurs masses volumiques mesurées puis analysées par diffusion des rayons X (SAXS) en solution diluée de toluène (fig. 1).

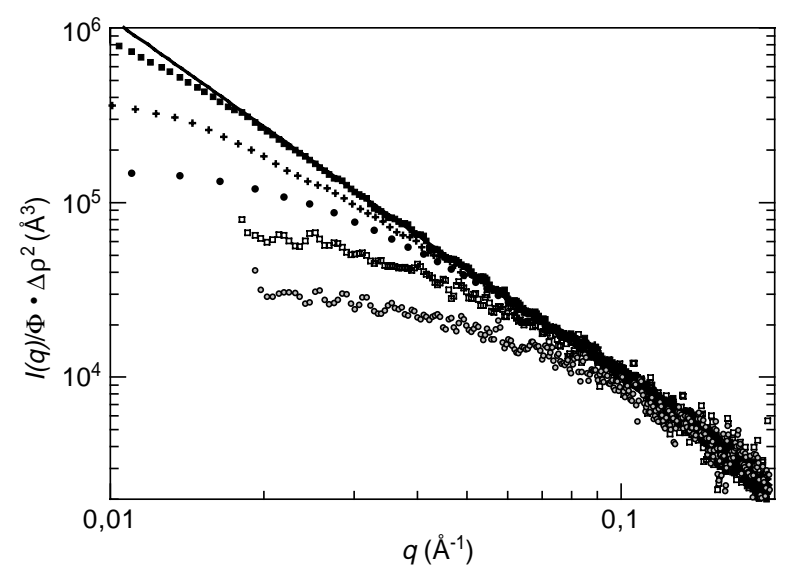

Figure 1

Spectres SAXS des asphaltènes centrifugés 1 (cercles ouverts), 2 (carrés ouverts), 4 (cercles pleins), 5 (croix) et culots (carrés pleins). Le trait est l'ajustement de l'équation (1) avec $d=2,06$ sur le spectre de la fraction culot.

SAXS spectra of centrifuged asphaltenes 1 (open circles), 2 (open squares), 4 (crosses) and base fraction (full squares). The full line is the adjustment of Equation (1) with $d=2.06$ on the base fraction spectrum.

\section{RÉSULTATS}

\subsection{Structure et polydispersité avant le seuil de floculation}

La forme des spectres de diffusion se décompose en un régime de Guinier aux petits angles, suivi d'un domaine de $q$ d'autant plus étendu que les asphaltènes sont fortement agrégés, sur lequel l'intensité diffusée décroît selon une loi de puissance de $q$ :

$$
I \approx q^{-d}
$$

L'exposant $d$ est égal à 2,06. De même, en utilisant la formule de Zimm [21] sur le régime de Guinier [22] des spectres :

$$
\frac{\Delta \rho^{2} \Phi}{I(q)}=\frac{N_{A} m_{v}}{M_{m}}\left(1+\frac{q^{2} R_{G}^{2}}{3}\right)
$$

où $\Delta \rho^{2}$ est le contraste et $N_{A}$ le nombre d'Avogadro, nous mesurons la masse molaire $M_{m}$ et le rayon de giration $R_{G}$ des asphaltènes reliés par la loi de puissance :

$$
M_{m} \propto R_{G}^{D}
$$

dont l'exposant est $D=1,99$. Nous avons pu déterminer la distribution en masse des agrégats d'asphaltènes dans le toluène. Sur l'intervalle de masse que nous avons étudié, le nombre $N$ d'agrégats d'asphaltènes de masse $M_{m}$ suit une loi de puissance :

$$
N\left(M_{m}\right) \propto M_{m}^{-\tau}
$$

avec $\tau=1,66$ assez proche de 1,5. Cette forme de polydispersité permet de relier les exposants $D$ et $d$ à la dimension fractale $D_{f}$ des asphaltènes : $D=d=D_{f} \approx 2[23,24]$.

Les asphaltènes en solution dans un bon solvant sont des agrégats fractals. Leur structure ouverte et solvatée occupe un important volume. Elle est compatible avec un modèle d'agrégation RLCA.

\subsection{Structure au-delà du seuil de floculation}

Nous avons enregistré les spectres de diffusion des neutrons (SANS) d'une solution diluée $(\Phi \approx 0,015)$ d'asphaltènes Safaniya dans un mélange de toluène et de $54 \%$ d'heptane, donc très proche du seuil de floculation de ces asphaltènes (seuil $\approx 56 \%$ ). La forme des spectres de diffusion des neutrons change brutalement au passage du seuil de 
floculation (fig. 2). Un signal très intense apparaît, obéissant à l'équation (1) avec $d=3,37$ aux petits angles, qui correspond à la formation rapide de structures compactes (dont la dimension fractale est proche de 3 ) de très grande taille $\left(R \geq 1 / q_{\min } \approx 500 \AA\right)$. Après quelques heures, les asphaltènes sédimentent. Ils sortent ainsi du faisceau de neutrons, et ne contribuent plus au signal diffusé aux très petits angles.

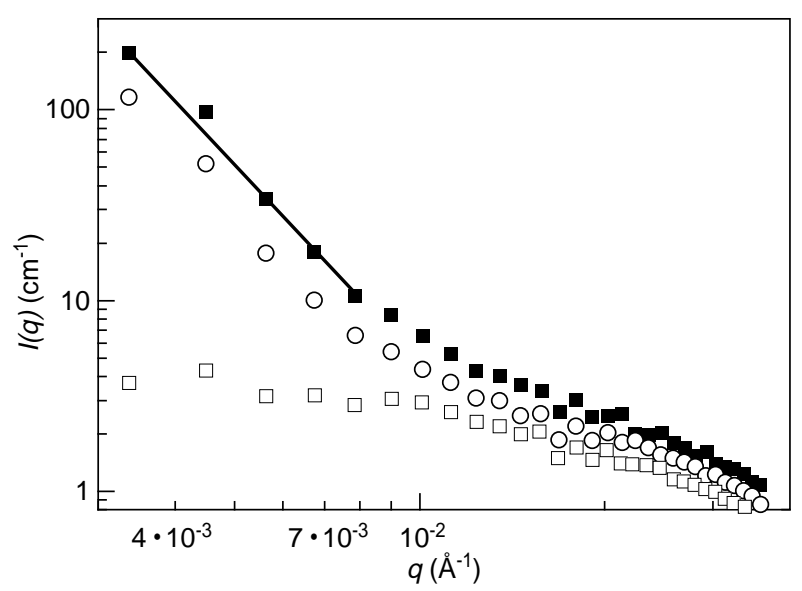

Figure 2

Spectres SANS d'asphaltènes Safaniya dans un mélange de $54 \%$ d'heptane et de $46 \%$ de toluène deutérés, mesurés pendant les 25 premières minutes (cercles), au bout de $5 \mathrm{~h}$ (carrés pleins), et au bout de 10 h 40 (carrés vides) après l'ajout d'heptane. Le trait est l'ajustement de l'équation (1) avec $d=3,57$ sur le premier spectre.

SANS spectra of Safaniya asphaltenes in a mixture of $54 \%$ heptane and $46 \%$ deuterated toluene measured during the 25 first minutes (full circles), at the end of $5 \mathrm{~h}$ (full squares), and at the end of $10 \mathrm{~h} 40 \mathrm{~min}$ (open squares) after the addition of heptane. The full line is the adjustment of Equation (1) with $d=3.57$ on the first spectrum.

Nous avons suivi, par diffusion dynamique de la lumière, la cinétique d'agrégation d'asphaltènes $K(\Phi=0,01)$ dans un mélange de $52 \%$ d'heptane et $48 \%$ de toluène qui correspond au seuil de floculation pour ces asphaltènes. L'évolution de la taille des asphaltènes en fonction du temps (fig. 3) est tout à fait compatible avec les structures ouvertes et solvatées décrites précédemment. Les valeurs expérimentales s'accordent parfaitement avec les prédictions du modèle RLCA (une exponentielle croissante du temps, représentée par le trait plein sur la figure 3) dans les premiers temps de l'agrégation.

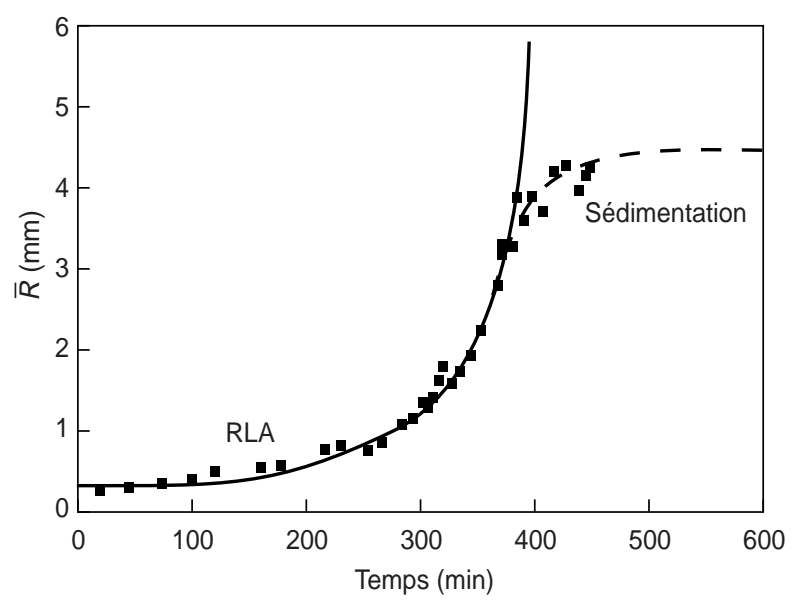

Figure 3

Cinétique d'agrégation d'asphaltènes $K(\Phi=0,01)$ dans un mélange de $52 \%$ d'heptane et de $48 \%$ de toluène. Le trait plein est l'ajustement d'une exponentielle croissante sur les mesures. Le trait pointillé sert à guider les yeux.

Asphaltene aggregation kinetics $(\Phi=0.01)$ in a mixture of $52 \%$ heptane and $48 \%$ toluene. The full line is the adjustment of an increasing exponential on the measures. The dotted line is used as an eye guide.

Puis, la taille des agrégats tend vers une valeur constante (proche de $5 \mu \mathrm{m}$ ). Nous attribuons cet écart aux prévisions du modèle RLCA à la compaction de la structure asphalténique. Nous constatons que la sédimentation des asphaltènes est une conséquence directe de cet effet de compaction. En effet, compte tenu de la viscosité du milieu et des différences des masses volumiques entre les asphaltènes et le solvant, la vitesse importante de sédimentation des asphaltènes (jusqu'à $1 \mathrm{~cm} / \mathrm{min}$, visible à l'œil nu) n'est accessible qu'à une structure compacte, et non pas solvatée.

\subsection{Les étapes de la floculation}

Le passage du seuil de floculation correspond à la séparation des asphaltènes en deux phases. La phase solvatée existe déjà avant le seuil ; la phase compacte apparaît au seuil de floculation. Cette seconde phase est généralement appelée floculée ou insoluble. Au fur et à mesure de la baisse de la qualité du solvant au-delà du seuil de floculation, la proportion d'asphaltènes floculés croît au détriment de la phase soluble. Dans un excès d'heptane (30 volumes, pour la norme AFNOR T60-115), tous les asphaltènes précipitent. Leur structure peut être observée dans la poudre d'asphaltène, dont le spectre de diffusion des rayons $\mathrm{X}$ aux petits et très petits angles (fig. 4) est caractéristique d'une structure dense. 


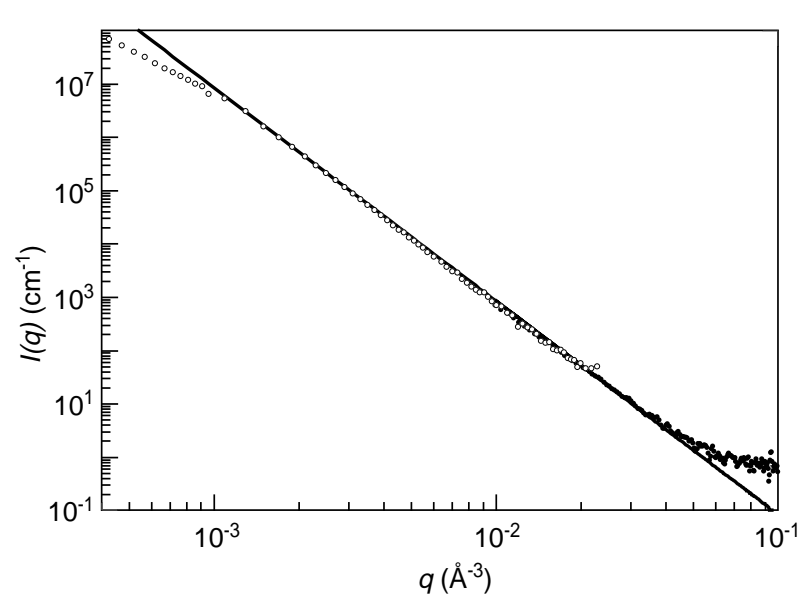

Figure 4

Spectres de diffusion USAXS (cercles vides) et SAXS (cercles pleins) de la poudre d'asphaltène. Le trait est l'ajustement de l'équation (1) avec $d=4$.

USAXS (empty circles) and SAXS (full circles) scattering spectra of asphaltene powder. The full line is the adjustment of Equation (1) with $d=4$.

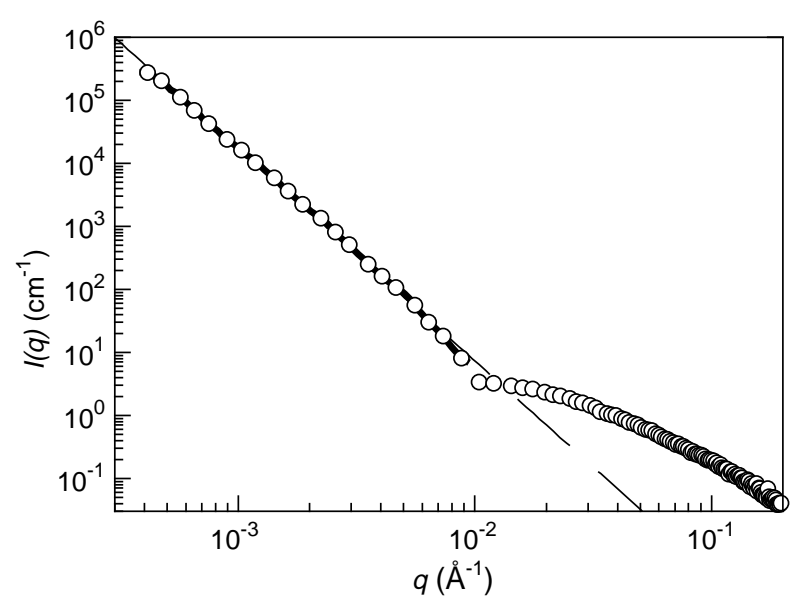

Figure 5

Spectres de diffusion des rayons X (SAXS et USAXS) du RSV Safaniya (cercles). Le trait est l'ajustement de l'équation (1) aux très petits angles avec $d=3,37$.

SAXS and USAXS X-ray scattering spectra of the Safaniya $V R$ (circles). The full line is the adjustment of Equation (1) in the very small angle region with $d=3.37$.
L'intensité diffusée par la poudre suit la loi de puissance (1) sur un domaine de vecteur de diffusion qui s'étend de $q_{\text {min }}=10^{-3} \AA^{-1}$ à $q_{\max }=4 \cdot 10^{-2} \AA^{-1}$. La pente $d=4$ du spectre indique non seulement que la structure est compacte sur l'échelle de taille correspondante (entre 25 et $1000 \AA$ A ) mais aussi que la surface des «grains» d'asphaltènes est lisse jusqu'à des tailles typiquement de l'ordre de $25 \AA$.

\subsection{De l'échantillon synthétique à un fluide réel}

L'étude d'asphaltènes en solution dans des solvants modèles, comme les mélanges de toluène et d'heptane, constitue une simplification expérimentale du système complexe qu'est le fluide pétrolier, simplification qui se justifie a posteriori par la reconnaissance de similarités entre les deux systèmes. Le fluide industriel que nous avons choisi est le RSV Safaniya dont la très forte viscosité $\left(\eta \approx 10^{7} \mathrm{~Pa} \cdot \mathrm{s}\right.$ à température ambiante [25]) interdit l'éventuelle sédimentation des asphaltènes floculés. Nous avons enregistré les spectres de diffusion SAXS et USAXS du RSV Safaniya (fig. 5). Ils se décomposent en deux parties : aux petits angles, la courbure du spectre correspond aux agrégats solvatés d'asphaltènes solubles ; aux très petits angles, l'intensité diffusée suit l'équation (1) avec $d=3,37$, caractéristique des structures compactes d'asphaltènes floculés qui restent en suspension dans le fluide du fait de la viscosité importante du milieu.

\section{CONCLUSION}

Les mesures par diffusion de rayonnement permettent de caractériser l'évolution de la structure des asphaltènes en fonction de la qualité de leur solvant. À l'approche de la floculation, des structures ouvertes et solvatées typiquement nanométriques croissent lentement. La cinétique d'agrégation est alors compatible avec un modèle d'agrégation limité par la réaction RLCA. Nous constatons que la floculation correspond à l'apparition de structures denses de très grande taille (micrométriques). La proportion d'asphaltènes dans la phase floculée augmente ensuite avec l'ajout d'heptane. Dans un solvant peu visqueux (comme les mélanges de toluène et d'heptane), ces agrégats compacts et de grande taille peuvent sédimenter.

Il est fréquemment considéré que l'étude des asphaltènes dans un solvant connu, comme le mélange de toluène et d'heptane, est une simplification justifiée du système industriel. Nous interprétons le spectre de diffusion des rayons X du RSV Safaniya, très intense aux petits angles, comme celui d'asphaltènes floculés. Aussi, la qualité de solvant des malthènes du RSV Safaniya ne serait pas suffisante pour empêcher la floculation d'au moins une partie des asphaltènes. Seule la viscosité importante du RSV Safaniya permet de conserver les asphaltènes floculés en suspension. 


\section{REMERCIEMENTS}

Nous sommes particulièrement reconnaissants envers P. Courtin et J. Lemerle du Laboratoire de chimie des polymères inorganiques de l'université Pierre-et-Marie-Curie de Paris pour la réalisation des séparations d'asphaltènes par ultracentrifugation. Nous souhaitons également remercier J.-N. Roux, D. Broseta et D. Espinat pour les discussions que nous avons échangées.

\section{REFERENCES}

1 Sheu, E.Y. et Mullins, O.C. (1995) Asphaltenes, Fundamentals and Applications, Plenum Press.

2 Yen, T.F., Erdman, J.G. et Pollack, S.S. (1961) Analytical Chemistry, 33, 1587-1594.

3 Thiyagarajan, P., Hunt, J.E., Winans, R.E., Anderson, K.B. et Miller, J.T. (1995) American Chemical Society, Energy and Fuels, 9, 829-833.

4 Nielsen, B.B., Svrceck, W.Y. et Mehrotra, A.K. (1994) Ind. Eng. Chem. Res., 33, 1324-1330.

5 Yudin, I.K, Nikolaenko, G.L., Gorodetsky, E.E., Kosov, V.I., Melikyan, V.R., Markhashov, E.L., Frot, D. et Briolant Y. (1996) $4^{e}$ Symposium international sur l'évaluation de la mouillabilité des réservoirs et son effet sur la récupération du pétrole, Montpellier, 11-13 sept.

6 Yudin, I.K., Nikolaenko, G., Gorodetskii, E.E., Markhashov, E.L., Frot, D., Briolant, Y. et Anisimov, M.A. (1998) J. Petroleum Science \& Technology, 16, 395-414.

7 Anisimov, M.A., Yudin, I.K., Nikitin, V., Nikolaenko, G., Chernoustan, A., Toulhoat, H., Frot, D. et Briolant, Y. (1995) J. Phys. Chem., 99, 9576-9580.

8 Raghunathan, P. (1991) Chem. Phys. Lett., 182, 331-335.

9 Janardhan, A.S. et Mansoori, G.A. (1993) Journal of Petroleum Science and Engineering, 9, 17-27.
10 Liu, Y.C., Sheu, E.Y., Chen, S.H. et Storm, D.A. (1995) Fuel, 74, 1352-1356.

11 Ravey, J.C. et Espinat, D. (1990) Progress in Colloid \& Polymer. Science, 81, 127-130.

12 Sheu, E.Y., Liang, K.S., Sinha, S.K. et Overfield, R.E. (1992) J. Colloid Interf. Sci., 153, 399-410.

13 Lin, M.Y., Lindsay, H.M., Weitz, D.A., Ball, R.C., Klein, R. et Meakin, P. (1989) Fractals in the Natural Sciences, in Fleischmann, M., Tildesley, D.J., Ball, R.C. (eds.), Princetown Paperbacks, 71-87.

14 Broide, M.L. et Cohen, R.J. (1990) Phys. Rev. Lett., 64, 2026-2029.

15 Broide, M.L. et Cohen, R.J. (1992) J. Colloid Interface Sci., 153, 493-508.

16 Weitz, D.A., Huang, J.S., Lin, M.Y. et Sung, J. (1984) Phys. Rev. Lett., 53, 1657-1660.

17 Meakin, P.P. et Family, F. (1987) Phys Rev., B 31, 564.

18 IFP-Sematech, DL 135-45 (19) Granulomètre submicronique pour milieux noirs et/ou concentrés, réalisé en collaboration avec la société Sematech dans le cadre de l'action PME/PMI.

19 Lambard, J. et Zemb, T.A (1991) J. Appl. Cryst., 24, 555-561.

20 Lake, J.A. (1967) Acta Cryst., 23, 191-194.

21 Zimm, B.H. (1948) J. Chem. Physics, 16, 1093-1116.

22 Guinier, A. et Fournet, G. (1955) Small-Angle Scattering of $X$-rays, John Wiley.

23 Auvray, L. et Auroy, P. (1991) Neutron, X-ray and Light Scattering, Lindner, P., Zemb, Th. (eds.), 199-221.

24 Teixeira, J. (1988) J. of Applied Crystallography, 21, 781785.

25 Guille, V. (1996) Étude de la structure des solutions d'asphaltènes et d'un résidu sous vide Safaniya, Thèse, université de Nancy I.

Manuscrit définitif reçu en mars 1999
Rédacteur en chef : R. PELET Réalisation : GraphiDoc 92852 Rueil-Malmaison Cedex - France Imprimerie JOUVE 75001 PARIS NNo d'impression : 278999 P - Février 2000 Éditeur : $n^{\circ} 244$ CPPAP : n 59260 IMPRIMÉ EN FRANCE 